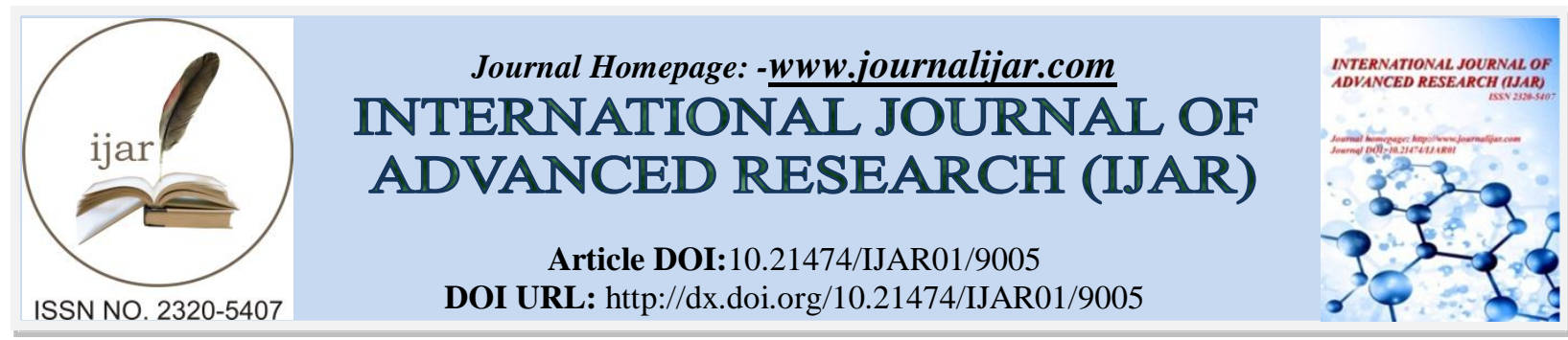

RESEARCH ARTICLE

\title{
WHAT CAUSES THE HUMAN LANGUAGE TO BE DIVERSE?.
}

Farman Zeynalov.

Azerbaijan University of Languages Baku, Azerbaijan.

\section{Manuscript Info}

\section{Manuscript History}

Received: 02 March 2019

Final Accepted: 04 April 2019

Published: May 2019

\section{Key words:-}

universe, languages, isomorphism, rhythm, system, structure, distribution, variation, matter, property, relation, natural factors, energy or heat, uneven, geographical areas, unconsciousness.

\begin{abstract}
This article deals with the factors which cause the human language to be diverse. The study of linguistic literature shows that linguists are much more interested in studying both universality and diversity characteristics of the human language which are of paramount importance from theoretical, practical and linguo-cultural point of view. Thus, the common and specific characteristics of the human language, to our mind, become the major aim and basis of linguistic theory of today.

As D.Crystal writes: "Why study languages?" or Why study language? Perhaps it ought to have been "language". For once again I must emphasize that the main task of the linguistic scholar is not to improve the language teaching situation..... etc.: his task is basically to study and understand the general principles upon which all languages are built. What are the "design features" of human language? This is his prime concern. How far can we define the universal characteristics of language? Or, putting this another way, "What are the differences between languages? How can we describe and classify these? and How far are they fundamental? What concepts do we have to develop before we can begin to talk about language at all'.(D.Crystal, 1977).

In this respect we hope that this article may throw some light on the questions mentioned above.

Our aim, accordingly, is to study the diversity of human language and to discover the natural factors which cause the human language to have different structures and properties.
\end{abstract}

Copy Right, IJAR, 2019,. All rights reserved.

\section{Introduction:-}

The theoretical background of the article is based on some considerations which are underlined in the works of distinguished linguists, such as J.Herder, V.von Humboldt, W.D.Whitney, H.Steintal, W.Wundt, E.Sapir, etc.

F.de Saussure`s dichotomic (language and speech), as well as system and structural approach to language, gave rise to the investigation of language in relation to other sciences, such as biology and geology, etc. In this respect the problem of isomorphism between language and genetic code has remained in the interest of R.Jacobson for a long time. (Jacobson, 1970)

The connection of language and geology was underlined by the participants of the conference dedicated to the problem of "Language and Earth" held in Bad Homburg (Germany) in 1989 (Naumann B.P., et al., 1992). It was 
revealed that there is structural isomorphism between the structure of crystals and the ornaments of ancient works of artifacts. (MammadovKh, 1981). Furthermore, it was revealed that there is structural isomorphism and functional similarity between language and crystals. (F.Zeynalov, 1999)

Thus, having assumed the above-mentioned considerations as a basis, we may say that isomorphic instances happen in the form of unconscious way not depending on the will or wish of human beings.

\section{Research Methodology:-}

In the article we have used direct observation, structural isomorphism, comparative linguistic description, transformation, logical-heuristic methods.

\section{Scope of study}

The investigation of the linguistic literature shows that there are various approaches and theories on the origin of human language, on the one hand, (Fromkin, Rodman. 1988) and the diversity of human language, on the other hand. Among them, J.Herder's considerations, who accepted the Cartesian rationalist position, seem to be convincing. According to Herder, language ability is innate and is an essential part of human nature. So it was neither invented nor handed down as a gift. He drew on the universality of all human languages as an argument to justify a monogenetic theory of the origin of human language. He put forth the monogenetic theory to explain why languages, despite their diversity, have universal common properties. (Fromkin, Rodman, 1988) Though the monogenetic theory was not widely accepted at that time, and in contemporary linguistics, however, American linguists on the basis of experiments have recently asserted that all the languages of the world have common roots $(2016,113(7))$.

It should be noted that the beliefs in the diversity of human language are found not only in the Tower of Babel legend, in the myths of other peoples, in monogenetic and polygenetic theories, as well as in the works of distinguished linguists. According to V. von Humboldt the diversity of human language is closely connected with a people's soul or spirit. W.D.Whitney related the diversity of languages to the manner of thinking or mental properties of a people. Other scholars, such as H.Steintal, W.Wundt, and E.Sapir, reasoned their cases, i.e. diversity of human languages, as the psychological properties of a people. Among these approaches, concerning the diversity of human languages V. von Humboldt's view was widely accepted, all the same, as "language is the soul of a people". V. von Humboldt stated that the structure of a language expresses the inner life of its speakers. Man lives with the world about him, principally, indeed exclusively, as language presents it (Farb P., 1977). The case for a close relationship between language and reality was stated by E.Sapir. To him, human beings do not live in the objective world alone, nor alone in the world of social activity... The fact of the matter is that the "real world" is to a large extent built upon the language habits of the group.

No two languages are ever sufficiently similar to be constructed as representing the same social reality (Farb P., 1977).

The relation of language to other sciences, namely, to biology was underlined by R.Jacobson. It was revealed that genetic code resembles linguistic code from structural and functional point of view, and it is not accidental that at the very beginning of deciphering genetic code, molecular genetics began to borrow linguistic notions and terms. At the same time in linguistics there are cases when it borrows notions and terms from molecular genetics, too. The structural isomorphism between two different information systems raises the question concerning the nature and origin of the systems, and the reasons of similar structural isomorphism. In this respect, the argument between R.Jacobson, a linguist, and F.Jacob, a biologist genetist is very characteristic. The essence of their argument is whether the structural isomorphism between genetic and linguistic codes is purely external, arising as a result of structural similarity of two different similar systems, which perform informative functions, or this isomorphism is a result of phylogenetic construction of the linguistic code. R.Jacobson maintains the second view, and F.Jacob supports the first one. Jacobsonian understanding of the structural isomorphism of genetic and linguistic codes presupposes evolutionary process imitating its structural principles, which are realized on condition of unconsciousness (GamkralidzeTh.V, 1988).

The fact of unconsciousness is not only the fact that is expressed in phylogenetic process of mounting of structural mechanism of language on the model of genetic code, but it is also observed in creative activity of ancient craftsmen 
who created geometrical ornaments imitating the structural formation of crystal substances. This fact became known to science after studying the structure of crystal by the X-ray structural analysis method. It was revealed that Moris Escher (1920) and his predecessors in their works used unconsciously mathematical model of crystals, i.e. they arranged their ornaments on the plane just as nature arranges molecules in crystal substances (Kh.Mammadov, 1981). Similar facts can be found in "creative" activity of bees. Bees build their honeycomb in accordance with the structure of aroma or odour, i.e. directly imitating the chemical structure of aromatic carbohydrates, whose structure is a hexagonal geometrical figure, which is identical with the shape of the bee cells, i.e. both are in the form of hexagonal geometrical figures. The structural formation and principle arrangement of bee cells are much similar to crystal ornaments, whose cells recur periodically in a honeycomb with the same shape. A spider spins its cobweb in a shape of geometrical figures. The cobweb of the spider consists of the same rhythmically arranged geometrical figures, though they are different in dimensions which are also similar to crystal ornaments. Moreover, it was revealed that due to its many parameters, language behaves like natural objects, particularly like crystals which possess a certain rhythmic property reiterated periodically. Thus, owing to its system and structural nature, rhythm, structural differences, function, ability to carry and store information, language resembles crystals and genetic code (F.Zeynalov, 1999).

This similarity shows that the isomorphism of language with geological processes, rather crystals and genetic code, is not at the level of abstract notions: there are objective relations in their origins. Though the birth and formation of these systems emerged in various periods of historical development, they conditioned the birth of each other, i.e. they inherited their peculiarities from each other. Therefore, the linguistic code, the structure of language haveemerged naturally in the process of phylogenetic evolution in compliance with the structural principles and structural models which existed before them.

As the structural model of language has emerged after the birth of the aforementioned systems, i.e. crystal and genetic code, their structural principles and other properties are conspicuously observed in language. Thus, language remains a natural phenomenon in the chain of hierarchy of systems of the nature, a link in this chain with its own embryo, to material basis and trace (F.Zeynalov, 1999). F. de Saussure's definition of language runs: "Language is a system of signs expressing ideas". In this respect, language as a system should meet several requirements: the system should consist of lower or undersystems; the system should constitute a part of a supreme system. Language as a system has lower or undersystems, such as the system of phonemes, morphemes, lexemes, phrasemes and so on. The connection of language with living organism is a generally known fact, but the facts revealing similarity between language and geology were unexpected to the participants of the conference dedicated to the problem of "Language and earth held in Bad Homberg (Germany): elective affinities between emerging sciences of linguistics and geology (1989). Relying on a number of revealed facts the scientists tried to explain the history of earth and the origin of language and the reasons that cause geographical and language changes on the basis of the catastrophic theory. They analysed and discussed different points of view of specialists of the $18^{\text {th }}$ and $19^{\text {th }}$ centuries on the structural and integrity between language, geology, chemistry and biology. They accentuated an idea that scientific achievements in the field of physical sciences had played an important role for anthropology and linguistics. In the $19^{\text {th }}$ century the realistic picture of chronological periods of development of the earth was formed, and on the basis of this fact of geology, some linguists (Y.Adelung and J.Grimm) considered it to be their main task to explain the development of language via the development of all mankind but with some geographical limitations. But according to Proceedings of the National Academy of Sciences, the American linguists asserted that all languages of the world have common roots. They came to such a conclusion after the analysis of some basic and ordinary words of 80 languages of the world. Primitive tools stimulated development of speech.

In the course of experiment, linguists chose several words close in their meaning, then translated them into one of 80 languages. The choice was made at random. After that, using all the meanings of words, they were translated again. As a result, the specialists compared the sets of meanings and determined to what extent they are related or far from each other.

The scientists chose mainly ordinary words connected with basic things such as, for example, the subjects of nature, celestial bodies, the day time and others.

The analysis of these semantic relations made it possible to determine there exist in the world three specific linguistic "clusters". The first is represented by words which describe phenomena related with water. The second includes words designating solid objects, for example, mountains. The third is related to heavenly bodies. 
Having determined this, the linguists came to a conclusion that all languages of the world originate from one root...

The scientists explained the reason of linguistic distinctions of different peoples. In their opinion, the reason of this phenomenon is conditioned by climatic factors (2016), 113(7).

All the above-mentioned facts show that the links of human beings with the universe are much deeper and more virtual than our considerations, and in many cases these unobservable and virtual links emerge in various ways or manners unconsciously. It should be noted that the act of unconsciousness brings a series of questions: how does it happen that there is structural isomorphism in crystals, genetic and linguistic codes? How does it happen that the structural model of crystals, as geometric figures, appear in ornaments, designs and minor arts, as well as weaving of carpets, rugs? How does it happen that there is rhythm within crystals: at first sight they seem to be dead and inanimate, whose atomic molecular structure recurs periodically creating the imagination of endlessness. How does it happen that genetic code corresponds to crystals with it periodically recurrence of self-creation: how does it happen that the linguistic code corresponds to genetic code with it periodically recurrence of self-creation? How does it happen that bees make their cells and spiders their cobwebs in the shape of rhythmically arranged geometrical figures? How does it happen that when a baby is born, the first act that he or she performs is crying with high pitch voice? How does it happen that the first organ formed in the embryo of a baby is the ears? How does it happen that human language becomes diverse which originates from one root? How does it happen that various articulatory-acoustic sounds and sound clusters occur in the identical speech apparatus? How does it happen that different cultural properties are formed in the human race?

It should be noted that to create diversity in order to make economy of atoms, elements and phonemes of a system and substitute this minority with the structure, is the main principle of nature (Kh.Mammadov). To put it another way, structure is one of the main principles or ways of creating diversities from the finite number of elements of the systems. It should be noted that all the substances or matters that we can observe are built from atoms of more than hundred chemical elements. It is possible to construct all the words in all languages from about thirty five phonemes. The minority of elements or phonemes of a system leads to their universality, on the one hand, and makes it easy to produce or build different words, forms and endless word combinations and sentences, on the other hand.

\section{Factors causing language to be diverse?}

The well-known Azerbaijani scientist KhuduMammadov's works and achievements in the field of crystallochemistry were highly appreciated by great scientists (John Bernal, N.Belov) and gained recognition by famous crystallographers of the world. The words written by the great English scientist John Bernal in his book, who personally presented it to KhuduMammadov, "to build a bridge from inanimate nature to the animate one" are of great interest in this respect (N.Rzayev, 1995). KhuduMammadov was known as a famous scientist-researcher, strucrtural-crystallographer, crystallo-chemist, a teacher possessing wide knowledge in biology, maths, physics, history, art and literature. He was a man of the widest world outlook and the founder of the "Back Groundless Formation of Fine Forms" (BFFF) in applied art and the common principle in national art and culture. (Mammadov, 1981)

On the grounds of comprehensive researches, KhuduMammadov arrived at some valuable conclusions, which throw light not only on applied art, let alone crystallochemistry and crystallography, but also on various branches of science, including linguistics. Here are some of them: whatever human beings create in their life activity, namely, in art and culture, etc., their prototypes do exist in nature, and human beings cannot have created such a thing the prototype of which is not found in nature. Human beings utilize the principles of conformation of nature and approach them in ideals; nature used its own richest principles in the structure of human beings, and they can only utilize these principles; the art modeling of general regularities of nature, first of all, is realized on the level of psychical processes, which are composed or built on the basis of the physical and chemical laws; the various branches of national art and culture, which are inherent in peoples' history are linked to one root and they all originated from one source (Kh.Mammadov, 1981). From this point of view, the diverse thoughts or ideas, which were expressed, during various periods of the development of languages, by scientists and philosophers on close relation between language and a people's soul or spirit (V. von Humboldt), the manner of thinking or mental peculiarities (W.D.Whitney), psychology (H.Steinthal, W.Wundt, E.Sapir and et al) art and culture, literature and history find visually its reflection and confirmation in KhuduMammadov's researches today, who determined the 
common features between two kinds of art examples, both visible and invisible, and discovered the common principle existing among various branches of national art and culture. He found out similarities in the structures of art examples, namely the similarity between melorhythmographic structure of the Azerbaijani mugams, folk songs, dance music and patterns of designs forming ornaments in Azerbaijani carpets, klims (rugs), etc, whose melorhythmographic structures in the form of ornaments are traced in the art examples (Kh.Mammadov, 1981).

In this connection one can ask oneself a question: what lies behind the common features or principles, which are traced inherently in people's art and culture. Some people take for granted that common features or similarities might be due to the accident and the others - to language.

Taking for granted that the common principle in people's art and culture is due to peculiarities of the national language, it seems to us, however, that there is one more natural reason for this: whose influence causes languages to be diverse from the viewpoint of articulatory-acoustic features, phonotactic and rhythmic arrangemnets, as well as word order and grammatical structure is the rhythm of nature. As it is known, rhythm is one of the universal properties of nature. So, the rhythm of nature is not stable and even everywhere on earth. It varies from continent to continent, from country to country, from region to region, even from village to village. The variations of the nature's rhythm in various geographical territories or areas are based on the energy and heat of the sun, whose heat is distributed unevenly around the earth. So the uneven distribution of the sun's heat around the earth creates unstable rhythm, which forms uneven rhythmic arrangements or structural patterns in various areas, what, in its turn, influences or affects the articulatory-acoustic features of the sounds of speech, phonotactic, and rhythmic arrangements of a certain language, as well as its grammatical structure. Having taken the above-mentioned viewpoints made by KhuduMammadov, as a basis or postulates, our thesis can be substantiated by the following real facts: It is known to science that the prototype of what human beings create does exist in nature. So, at present it has been found out that the prototypes (or forms) of most geometrical figures exist in the structure of crystal substances, and the forms of various multiangular figures exist in the chemical structure of substances. Many of the rhythms, which men put into music and poetry, may be found in nature, such as drumming of the rain, beating of the rain and waves; singing of a canary and other birds, etc. Futhermore, the metrical measure of verses is imitated from nature, i.e. the syllabic meter is taken from the pace of a horse; Aruz meter-form various temporal paces of a camel and syllabo-tonic meter from the pace of a lynx. As O'Connor writes: "The sounds of speech are all round us. We use them, we enjoy and suffer from them and in general we know remarkably little about them”. (O’Connor J.D., 1977). The body of various musical instruments can also be found in nature. For instance, the body of a Lyre is U-shaped, which is like the tail of a lyre-bird; the body of a "Kamancha" is spherical (ball) - "Tar" and "Saz" are pear-shaped. The cells of bees are sixangular figure-shaped; the nest of an oven-bird is oven-shaped, etc. Various physical and chemical phenomena existing in nature created their own organs in human beings. Thus, light created the eyes; sounds- the ears and the vocal chords; or folds rhythm- the heart; metabolism - the stomach, liver, etc.

To put it another way, the material substance of language, that is the voice, noise are transformed from the nature to human organism. This finds its mere assertion in the facts that the first organ formed in the embryo of a baby is the ears, and when the baby is born the first act that he or she performs is crying with the high pitch voice.

So, if the prototype of the imitative art and culture created by human beings exists in nature (the crystal ornaments or geometrical designs, plants, fruits, flowers and animal figures); then, the structural forms and rhythmic arrangements of verses as an art modeling or patterns of design also exist in nature, whose material body is reflected in the forms of speech sounds. Moreover, if nature used its own richest principles in the structure of human beings, then on the contrary, it can be said, that what they use as communicative and moral or aesthetic - emotional means, i.e. language, poetry, music, etc., their prototypes, structures and rhythmical arrangements or patterns exist in nature, too. Such regularity finds its own physical analogy in the phenomenon of Faraday's electromagnetic induction. From this stand-point, Ch. Hockett's ideas and considerations are of great interest: "Sound change does go on because of noise. Only in a permanently noiseless universe would sound change cease; in a permanently noiseless universe there would be no events, hence, no information, hence nothing to talk with" [Hockett, 1965]. On the basis of this elementary physical principle, we may say that sounds of speech (language) containing those prototypes exist in nature; nevertheless, if the art modeling of general regularities of nature is composed on the basis of physical and chemical laws, then, the structure and verse forms of each language and their rhythmical arrangements, as an art modeling and as a means of it, are also composed on the basis of physical and chemical laws, i.e. the regularity of nature whose first material and inherent structural element is rhythm, which is reflected in the forms of speech sounds and their rhythmic arrangements in words, word combinations and sentences. Furthermore, it was found out 
that there is correspondence between the structure of substances and their properties. The structure often determines the properties of substances and at the same time the property affects the structure as well. Substances with similar chemical composition confirm identical-shaped crystals when hardened or crystallized. Though the chemical composition of diamond and graphite is the same, their structures are quite different and their properties are different, too. Thus, diamond is the hardest of all elements and graphite is the softest. This is due to their structure. There is correspondence between the structure of albumous substances and their properties. It is known that life is the living form of albumous substances and nucleic acids, and as life develops not in straight lines but always falls and rises, i.e. in a spiral shape, therefore the structure of albumous substances, as well as the structure of the nucleic acid, is spiral-shaped. But when albumen is denatured, the structure is broken, too. The structure and property correspondence is observed in the activity of living organisms. As it was mentioned before, bees build their cells in a honey comb in accordance with the chemical structure of aroma or odour; therefore there is a similarity between the shape of bee cells and the chemical structure of aromatic carbonhydrates, whose structure is a sixangular geometrical figure, which coincides with the cells of bees in a honey comb; both of them are in the form of a sixangular geometrical figure. In accordance with this isomorphism, the different kinds of birds always build their own kinds of nests, each with its own structural and rhythmical shape. For instance, a south American oven-bird's nest is always oven-shaped. It was also found out that there is a close connection between the biological rhythm of the human organism and the rhythm of the work of art. The experiments show that there is much more similarity between the rhythm of poetry, music and the biological rhythm. So, the more similarity in character and structure between the two rhythms (poetry, music and biological) the better aesthetic and emotional delight they arouse. Therefore, the folk songs, dance music and lyrical verses arouse much more aesthetic emotional delight in man and woman whose rhythms (biological) are isomorph in character and structure with the rhythms of their folk songs, dance music and poetry. The formation of similar rhythms (musical, poetical and biological) in character and structure is based on interrelation between the exogenous and endogenous rhythmic processes which are realized and formed by environmental factors of the areas, namely by the temperature (heat), air, water, light, and mainly by foodstuff which is grown and produced on the soil of that geographical territory or area. Although the rhythm of nature is not directly observed and seen like aroma or odour of flowers, air, gas, the thought but it is felt and heard in the forms of different linguistic structures such as sounds, combinations of sounds, syllables, words, allocations, sentences (utterances), verse lines, intonation groups, texts etc. Rhythm comes from movement. The movement, in its turn, is based on a certain energy or heat. All kinds of motion in substances, plants, and living organisms can be accelerated with the rise of temperature or arrested with lowering of it, which affects the character and structural composition of the rhythm in areas. It is already believed that racial differences in human beings are formed as a result of environmental factors whose morphological structure mainly depends on the climate and the geographical territories they inhabit historically.

In accordance with it, it can be said that the environmental factors with their rhythmical peculiarities may also affect the ability of the movement of the organs of speech, whose ability in forming certain speech sounds, as well as in arranging them in words, may be increased or restricted. This is reflected in the acoustic properties (pitch, intensity, length, timbre) of speech sounds, as different positions or articulations produce different acoustic effects, i.e. different speech sounds. So the formation of various or diverse speech sounds and acoustic sound features is due to movements and configurations of the speech organs which are regulated by the exogenous and endogenous rhythmic processes. Speech sounds, as well as their arrangement in words and sentences, have a number of physical properties which can be measured, analyzed, and their quantities relation determined. Unlike other sciences, when studying the properties and alternations of the matter, physics utilizes and includes the physical quantities. The physical quantities, characterizing the properties of matter and the most important relations between the physical quantities, which are expressed by mathematical formula, reflect the regularities and laws of nature.

If it is so, then the articulatory-acoustic properties of speech sounds and their arrangement in words and sentences of a language, whose acoustic properties can be measured, analyzed and expressed in figures, and the relation between the physical quantities which reflect the regularities existing in nature, can be taken as reflection of the property of the rhythmical structure of the geographical territory or area. This viewpoint finds its support in the following citation: "it occasionally happens that a foreign student who acquires faultless pronunciation and even correct intonation, and one wonders what is that betrays his non-English origin. It is, in these circumstances, his faulty rhythm". Furthermore, examples of Shakespeare's poetry all show that while the English language may have changed to a certain extent in form and pronunciation and idiom, its speech rhythm has remained unaltered for three hundred and fifty years [Milne B.L., 1977]. 
Taking into consideration the propositions of molecular-kinetic theory, and all the above-mentioned facts, we may say that speech sounds and their chain in the speech continuum do not only mutually influence and depend upon one another, but also mutually influence and depend on the rhythm of environmental factors, i.e. language is not a closed system; like any other natural systems, language is an open system, too.

As it was mentioned before, in order to consider investigation of an object as a system, it should meet several requirements: the system should consist of lower or undersystem; the system should constitute a part of a supreme system. Language as a system has lower, or undersystem, such as system of phonemes, morphemes, lexemes, phrasemes, and so on. If language is a system, then in this case it should constitute a part of a supreme system. To our mind, the supreme system of language is the universe. From this point of view, W. Chafe's considerations on language are of great importance. To Chafe language is a set of habits relating its vocal sounds with the universe... it impinges on non-linguistic reality in two ways; the vocal sounds, on the one hand, and the universe of things talked about, on the other hand... the study of all potential properties is phonetics, while the study of all properties of the universe, that are talked about, is semantics. Language selects properties from phonetics and semantics which are combined by language in various arrangements, the study of which constitutes phonology. When phonetic properties are involved, and grammar, when properties belong to semantics. Phonological and grammatical habits are independent of each other, but the two are linked by other complex habits, the study of which is called morphophonemic (Chafe, 1962).

On the basis of the property and structure correspondence, we may also conclude that the common principles and peculiarities, which are traced in art and culture of a nation, are based on peculiarities of a national language, whose origin and patterns of phonotactic and phonorhythmomelodic structures are conditioned mainly by the rhythm of the geographical territory, which the people inhabited historically and live there nowadays. Thus, language (speech) is the production of a society, the members of which are considered to be biological and social mechanisms and actualizers of their language. However, its structural model, as well as the linguistic peculiarities of every language in the form of various structures, exist in nature, whose articulatory - acoustic peculiarities, phonotactic arrangement of sounds in words and rhythmic-melodic patterns are composed or formed in accordance with the physical and chemical laws, which characterize the rhythmical laws and the rhythmical peculiarities of a certain area.

Proceedings from all the above-mentioned considerations, the substantial study of the subject and analyzed issues, allow us to make the following inferences:

The articulatory-acoustic peculiarities of every language, as well as their phonotactic, rhythmic-melodic arrangements and patterns of designs, are reflections of the rhythmical structure of a certain geographical area and the sound changes, which occur diachronically in languages, and are mostly affected by the relative changing the rhythm of nature, as well as the changing rhythm of the areas.

The culture and art, as well as manner of thinking or mental peculiarities of a nation, are formed on the basis of rhythmical characteristics of a geographical area. To put it another way, language peculiarities of a people - both segmental and suprasegmental properties, are formed and transformed to the peoples' language in accordance with the rhythmical structure of the geographical territory where the people settled and lived there historically. So all the information belonging to a people's culture, art (music, folk songs and dance, music, etc.) and history are loaded in the process of phylogenetic development in their language and are coded. In this respect, the language of a people may be considered as the core or nuclear element of people's culture, art and historical evidence. To investigate or study of the aspects of a language, or a language as a whole, deeply and indetail, one can get virtual information, which exists in so called deep structures of people's culture, art, music, manner of thinking or mental characteristics. At the same time, it should be noted that the virtual information about a people are reflected not only in lexicalsemantic properties of a language, but also by rhythmic-melodic structure of verses, texts, musical properties of folk songs, etc., whose rhythmic-melodic arrangements or shape correspond to the ornaments and forms of minor arts created by the craftsmen of the people. So the rhythmic-melodic structure of a language, rather speech of a people, carries an important information about linguo-paleontological properties of people's culture and art. To put it another way, according to rhythmic-melodic structure of their speech, music, songs, etc., it is possible to determine to what people this or that art examples belong.

Furthermore, it is possible to classify languages according to their rhythmic-melodic structure and find out differences and similarities in their structure on the basis of degree of tonality, levels of register, range differences, 
etc. At the same time on the ground of rhythmic-graphical configurations or patterns of languages, it is possible to determine the rhythmic-melodic structure of a certain geographical area, as well as, to determine the distribution of degree of energy of a certain area and in this respect, to our mind, the rhythmic configuration or pattern of an area is just the reflection of the same rhythm which is transformed to diverse languages. So there is a rhythmic-melodic isomorphism between geographical areas and languages.

Thus, the assumption, leading to explanation of structural differences of languages, may be called a helio-rhythmic approach or theory. According to the helio-rhythmic theory, factors causing languages to be structurally (articulatory, that is their acoustic phonotactic, rhythmic-melodic features, etc.) diverse are the variations of nature's rhythm in various geographical territories, which are caused by the uneven distribution of the sun's heat (energy) around the earth. As the nature's rhythm varies, the different rhythmical variations form different rhythmical structures, which in their turn, are reflected in the place of articulation, acoustic properties and rhythmic-melodic patterns of languages. This viewpoint is based on the structure and property of mutual determination, in which the structure may be determined by property, or vice versa, at the same time any property or peculiarity cannot exist outside of its matter, and they always belong to certain objects or structures. According to the structure and property of mutual determination, we may say that different acoustic patterns of rhythmic-melodic structures of languages are the reflection of rhythmical peculiarities of different areas. The rhythmic-melodic peculiarities of a language form a certain system, which involves some information in its structure side by side with the words. The rhythmic-melodic structure of a language is able to carry information in its structure on history, art and culture of a people. So, by means of the rhythmic-melodic pattern of language, a historical settlement of a people may be determined on the basis of structural differences of rhythmic-melodic existence of the people's art and culture.

The attitude of individual persons to their homeland, language, music, art and culture, etc., may be also determined by the phonorhythmo-melodic peculiarities of their speech, as the speech of each national language carries or involves in a certain information from national-spiritual and moral state of individual persons, as well as from their inward world. Thus, we may say that language is like an insatiable sponge that absorbs all information, i.e. all the above-discussed factors, that surrounds humans of our planet, and one of the tasks of linguistic or phonologuistic investigation is to transform indefinite information into definite one, by means of computer experiments and electronic-acoustic analysis.

Whatever exists, be it a tiny ant, carrying his share to its ant-hill, or stars and galaxies billions and billions of light years away - is an integral rhythmic system, which as a whole, is moving, and its movement is fueled by the energy of the universe.

Everything that is visible and audible is manifested, forming evolutionally out of variation of the rhythmic system. The difference in the degree of distribution of energy in the universe varies the rhythmic system, and the inequality in the degree of distribution of energy is the main factor which forms diversity. Unequal distribution of energy varies the rhythm of geographical areas. This variation of the rhythmic system forms articulatory-acoustic shape of the phonetic elements and the combinatorics of the phonetic system of a human language, grammar structure, rhythmic and melodic peculiarities of speech.

Thus, the rhythmic system of the universe exerts influence on climate factors, and as a result, a peculiar rhythmic system of every geographical area is developed.

The rhythm of climate factors, in some cases, assimilating humans and their psychology, behavior, language, culture, art, genes etc., develops them in an unconscious way, in accordance with the variety of its rhythmic structure.

For this reason, psychology, language and behavior of nations, phonetic system, combinatorics, rhythmic and melodic peculiarities of their language, the variety of patterns of culture and art are manifested, having formed in different ways.

\section{References:}

1. Abdullayev S.A. (2013) Experience fuzzy linguistics, Baku

2. Chafe W.L. (1962) Phonetics, semantics and language. Language, 36(4), 335-344.

3. Crystal D. (1977) Linguistics. London: Penguin Books. 
4. Farb P. (1977) Word play. What happens when people talk.Caronet Books.

5. Fromkin V. and Rodman R. (1988). An introduction to language (4 ${ }^{\text {th }}$ ed.) $417-418$.

6. GamkralidzeTh.V. (1988) Jacobson and the problem of isomorphism between genetic and semiotic systems. Issues of linguistics (3) Moscow.

7. Hockett Ch. (1965) Sound Change. Language, 1(2), 185-204.

8. Jacob F. (1977) The linguistic model in biology. In: D.Armstrong and C.H.vanSchooneveld (Eds), Roman Jacobson. Echoes his Scholarship.

9. Jacobson R.O. (1970) Linguistics and its relation to other sciences. In: Main trends of research in social and human sciences. Part 1. Social sciences (pp.419-463). Paris: The Hague, Moaton.

10. Jacobson R.O. (1985) Selected works, Moscow.

11. Kurilovich Y. (1962) The notion of isomorphism. In: Outlines of Linguistics, Moscow.

12. Julla S.F. (1978). Linguistics and language ( $2^{\text {nd }}$ ed) New York.

13. MammadovKh. et al. (1981) Memories of ornaments, Baku.

14. Mammadov A.Y. (2008) Current issues in linguistics, Baku.

15. Milne B.L. (1977) English speech rhythm in theory and practice, London.

16. Naumann B.P., Plank F. and Hofbauer C. (1992). Language and earth: elective affinities between the emerging sciences of linguistics and geology. Amsterdam, Philadelphia: Benyamins.

17. O`Connor, J.D. (1970) Stress, Rhythm and Intonation. Madrid.

18. O`Connor, J.D. (1977) Phonetics. London.

19. Proceedings of the National Academy of Sciences of the United States of America (2016), 113(7).

20. Rajabli A.A. (2007). History of linguistics. Part I. Baku.

21. Rzayev N. (1995) The secrecy of Khudu`s Key. Baku.

22. Zeynalov F.H (1999) Language and reality: system and structural relations, Baku.

23. Zeynalov F.H. (2016) Universal properties of human language in the light of natural phonemena. In: International Journal of English Linguistics 6(1), 187-194. Canadian Center of Science and Education. 